\title{
A 2-nt RNA enhancer on exon 11 promotes exon 11 inclusion of the Ron proto-oncogene
}

\author{
HEEGYUM MOON $^{1}$, SUNGHEE CHO ${ }^{1}$, TIING JEN LOH ${ }^{1}$, JIANHUA ZHOU ${ }^{2}$, CLAUDIA GHIGNA ${ }^{3}$, \\ GIUSEPPE BIAMONTI ${ }^{3}$, MICHAEL R. GREEN ${ }^{4}$, XUEXIU ZHENG ${ }^{1}$ and HAIHONG SHEN ${ }^{1}$ \\ ${ }^{1}$ School of Life Sciences, Gwangju Institute of Science and Technology, Gwangju 500-712, Republic of Korea; \\ ${ }^{2}$ Jiangsu Key Laboratory of Neuroregeneration, Nantong University, Nantong 226001, P.R. China; ${ }^{3}$ Institute of Molecular \\ Genetics, National Research Council, I-27100 Pavia, Italy; ${ }^{4}$ Howard Hughes Medical Institute and Programs in Gene Function \\ and Expression and Molecular Medicine, University of Massachusetts Medical School, Worcester, MA 01605, USA
}

Received August 4,2013; Accepted October 14, 2013

DOI: 10.3892/or.2013.2835

\begin{abstract}
Ron is a human receptor for the macrophage-stimulating protein (MSP). Exon 11 skipping of Ron pre-mRNA produces the Ron $\Delta 165$ protein that has a deletion of a 49 amino acid region in the $\beta$-chain extracellular domain. Ron $\Delta 165$ is constitutively active even in the absence of its ligand. Through stepwise deletion analysis, we identified a 2-nt RNA enhancer, which is located $74 \mathrm{nt}$ upstream from the 5 ' splice site of exon 11, for exon 11 inclusion. Through double-base and single-base substitution analysis of the 2-nt RNA, we demonstrated that the GA, CC, UG and AC dinucleotides on exon 11, in addition to the wild-type AG sequence, function as enhancers for exon 11 inclusion of the Ron pre-mRNA.
\end{abstract}

\section{Introduction}

The RON receptor tyrosine kinase along with c-Sea, c-Met and Stk are members of the MET proto-oncogene family (1). The RON gene consists of 20 exons (2). RON protein is a $180-\mathrm{kDa}$ heterodimeric protein composed of a $40-\mathrm{kDa} \alpha$ chain and a $150-\mathrm{kDa} \beta$ chain linked by disulfide bonds (3). While the $\alpha$ chain contains the extracellular domain for ligand binding, the $\beta$ chain includes the intracellular part that contains a kinase domain and a transmembrane domain (4). These two chains are derived from the $180-\mathrm{kDa}$ precursor protein by a proteolytic cleavage (5). The macrophage stimulating protein (MSP) was

Correspondence to: Dr Jianhua Zhou, Jiangsu Key Laboratory of Neuroregeneration, Nantong University, 19 Qixiu Road, Nantong 226001, P.R. China

E-mail: jianhua@ntu.edu.cn

Dr Xuexiu Zheng and Dr Haihong Shen, School of Life Sciences, Gwangju Institute of Science and Technology, 1 Oryoung-Dong, Gwangju 500-712, Republic of Korea

E-mail: xuexiuzheng@gist.ac.kr

E-mail: haihongshen@gist.ac.kr

Key words: Ron, proto-oncogene, cancer, pre-mRNA splicing, exon 11 inclusion, cis-elements first identified as a ligand for Ron protein (6). MSP binds to the RON receptor to upregulate RON kinase activity, which leads to autophosphorylation on the tyrosine residues in the kinase domain and the C-terminal docking site (7-9). Activation of the RON receptor by MSP stimulates a large number of downstream intracellular pathways (10). Tumor formation and progression occurs when the accumulation and activation of receptor tyrosine kinases are abnormal (11). RON overexpression and activation induce tumor progression and invasive growth of certain types of epithelial tumor cells $(12,13)$. Alternative splicing of Ron pre-mRNA produces various protein isoforms (14). RON $\Delta 165$ protein, identified in gastric cancer cell line KATOIII, is generated by skipping of exon 11 (15). RON $\Delta 165$ does not undergo proteolytic processing and is retained intracellularly. Furthermore, uneven numbers of cysteine residues in RON $\Delta 165$ produces the RON oligomer. Therefore, RON $\Delta 165$ is constitutively activated without the binding of the MSP ligand. Abnormal accumulation of this isoform was found in some types of breast and colon cancer cell lines (16). Furthermore, overexpression of this splice variant can induce invasive growth and metastasis (15). Apart for the fact that ASF/SF2 induces skipping of exon 11 to control cell motility (16), the splicing mechanism of Ron exon 11 is not yet well understood.

Pre-mRNA splicing is a process in which introns are removed and then exons are ligated (17-19). The RNA sequences required for splicing are called splicing signals that include the $5^{\prime}$ splice site, $3^{\prime}$ splice site, branch point and polypyrimidine tracts (PPT) (20). In the alternative splicing procedure, different splicing signals are selected to produce multiple mRNA isoforms from a single gene through the numerous combinations of multiple exons (21). Alternative splicing is one of the critical mechanisms for gene regulation that generates proteomic diversity $(22,23)$. Abnormal regulation of alternative splicing causes a variety of human diseases including cancer (24). Alternative splicing is finely regulated by several cis-acting elements and trans-acting elements $(25,26)$. cis-acting elements are RNA sequences on pre-mRNA that function as either enhancers or inhibitors to regulate exon inclusion or skipping. Some cis-elements provide binding sites for SR proteins and hnRNP proteins to regulate 
splicing. Juxtaposed enhancers and inhibitors functionally antagonize each other $(27,28)$. Exon 11 inclusion of Ron premRNA is regulated by a juxtaposed enhancer and inhibitor on exon 12 (16). In the present study, we showed that exon 11 of Ron pre-mRNA also contains various cis-regulating elements for exon 11 inclusion. Specifically, a 2-nt RNA, located at 74 nt upstream from the 5 ' splice site of exon 11, functions as an enhancer for exon 11 inclusion. Through double base and single base substitution analysis on the 2-nt RNA, we demonstrated that the GA, CC, UG and AC dinucleotides on exon 11, in addition to the wild-type AG sequence, function as enhancers for exon 11 inclusion of Ron pre-mRNA.

\section{Materials and methods}

Construction of plasmids. The wild-type RON exon 10-12 sequences were amplified from human genomic DNA using RON10-HindIII-for and RON12-XhoI-rev primers (Table I) and cloned into HindIII and XhoI restriction enzyme sites of the pCDNA3.1 (+) vector. Every deletion and mutation construct was produced with overlapping PCR. All primers used for minigene constructs are listed in Table I.

Cell culture and transfection. MDA-MB-231 cells were grown in RPMI-1640 medium supplemented with $10 \%$ fetal bovine serum (FBS) at $37^{\circ} \mathrm{C}$ in a humidified $5 \% \mathrm{CO}_{2}$ atmosphere. Ron minigene transfection into MDA-MB-231 cells was carried out with polyethyleneimide (PEI) according to the manufacturer's protocol.

$R T-P C R$. Total RNA was extracted from the MDA-MB-231 transfected cells using RiboEx reagent (GeneAll, Korea) following the manufacturer's protocol. Total RNA $(1 \mu \mathrm{g})$ was reverse transcribed using oligo $\mathrm{dT}_{18}$ using ImProm-II ${ }^{\mathrm{TM}}$ reverse transcriptase (Promega, Madison, WI, USA) following the manufacturer's protocol. cDNA $(1 \mu \mathrm{l})$ was amplified by PCR using G-Taq polymerase (Cosmo Genetech, Seoul, Korea). RON minigenes were as following: RON10-forward (5'-CCTGGCTTTCGCTTCCTACC-3') and pCDNA-reverse (5'-CTAGAAGGCACAGTCGAGGCT-3'). GAPDH primer sequences were as following: GADPH-forward (5'-ACCACAG TCCATGCCATCA-3') and GAPDH-reverse (5'-TCCACC ACCCTGTTGCTGTA-3').

\section{Results}

Exon 11 contains various regulatory elements for exon 11 inclusion of Ron pre-mRNA. In order to identify the enhancer on exon 11 for exon 11 inclusion of Ron pre-mRNA, we performed mutagenesis analysis on exon 11. In the first step, we divided exon 11 into six parts $(11-1,11-2,11-3,11-4,11-5$ and 11-6) with the first five parts containing 20-nt RNA in each and the last part containing 17-nt RNA. The first and last parts are located $15 \mathrm{nt}$ apart from the $3^{\prime}$ and $5^{\prime}$ splicing sites of exon 11 (Fig. 1A). We produced deletion mutants for each part of RNA named as $\Delta 11-1, \Delta 11-2, \Delta 11-3, \Delta 11-4, \Delta 11-5$ and $\Delta 11-6$. We extracted RNA from the mutant minigene-transfected cells, and then performed RT-PCR analysis for Ron exon 11 splicing on each mutant. As shown in Fig. 1B, exon 11 splicing of $\Delta 11-2$ had the similar level of exon 11 inclusion as that of the
Table I. The primers used.

\begin{tabular}{|c|c|}
\hline Name & Sequences $\left(5^{\prime}-3^{\prime}\right)$ \\
\hline RON10-HindIII-for & ATGTTAAGCTTCCTGAATATGTGGTCCGAGAC \\
\hline RON12-XhoI-rev & CTTACCTCGAGCTAGCTGCTTCCTCCGCCACC \\
\hline$\Delta 11-1$-for & TATATTGGGCTGGGCTATCAACGTGACCGT \\
\hline$\Delta 11-1-$ rev & ACGGTCACGTTGATAGCCCAGCCCAATATA \\
\hline$\Delta 11-2$-for & GGCTGACTGTGTGGGGTGAGAGCTGCCAGC \\
\hline$\Delta 11-2-r e v$ & GCTGGCAGCTCTCACCCCACACAGTCAGCC \\
\hline$\Delta 11-3$-for & ACGTGACCGTGGGTGTTCCGGGGGGACATG \\
\hline$\Delta 11-3$-rev & CATGTCCCCCCGGAACACCCACGGTCACGT \\
\hline$\Delta 11-4$-for & AGCTGCCAGCACGAGCTGCCCCCTGCCCCC \\
\hline$\Delta 11-4-$ rev & GGGGGCAGGGGGCAGCTCGTGCTGGCAGCT \\
\hline$\Delta 11-5$-for & GGGGGACATGGTTGTTGCAGCTTGGCCAGG \\
\hline$\Delta 11-5$-rev & CCTGGCCAAGCTGCAACAACCATGTCCCCC \\
\hline$\Delta 11-6$-for & CCCTGCCCCCATCCCGGTGCCCCATTGCAG \\
\hline$\Delta 11-6-r e v$ & CTGCAATGGGGCACCGGGATGGGGGCAGGG \\
\hline$\Delta 11-3-1$-for & ACGTGACCGTGGGTGCCAGCACGAGTTCCG \\
\hline$\Delta 11-3-1-$ rev & CGGAACTCGTGCTGGCACCCACGGTCACGT \\
\hline$\Delta 11-3-2$-for & GGGTGGTGAGAGCTGTTCCGGGGGGACATG \\
\hline$\Delta 11-3-2-r e v$ & CATGTCCCCCCGGAACAGCTCTCACCACCC \\
\hline$\Delta 11-3-2(\mathrm{R} 2)$-for & CTGCCAGCACGTTCCGGGGGGA \\
\hline$\Delta 11-3-2(\mathrm{R} 2)-\mathrm{rev}$ & TCCCCCCGGAACGTGCTGGCAG \\
\hline UU-for & CTGCCAGCACGTTTTCCGGGGGGA \\
\hline UU-rev & TCCCCCCGGAAAACGTGCTGGCAG \\
\hline CA-for & CTGCCAGCACGCATTCCGGGGGGA \\
\hline CA-rev & TCCCCCCGGAATGCGTGCTGGCAG \\
\hline CU-for & CTGCCAGCACGCTTTCCGGGGGGA \\
\hline CU-rev & TCCCCCCGGAAAGCGTGCTGGCAG \\
\hline CC-for & CTGCCAGCACGCCTTCCGGGGGGA \\
\hline CC-rev & TCCCCCCGGAAGGCGTGCTGGCAG \\
\hline GA-for & CTGCCAGCACGGATTCCGGGGGGA \\
\hline GA-rev & TCCCCCCGGAATCCGTGCTGGCAG \\
\hline GU-for & CTGCCAGCACGGTTTCCGGGGGGA \\
\hline GU-rev & TCCCCCCGGAAACCGTGCTGGCAG \\
\hline GC-for & CTGCCAGCACGGCTTCCGGGGGGA \\
\hline GC-rev & TCCCCCCGGAAGCCGTGCTGGCAG \\
\hline UA-for & CTGCCAGCACGTATTCCGGGGGGA \\
\hline UA-rev & TCCCCCCGGAATACGTGCTGGCAG \\
\hline UC-for & CTGCCAGCACGTCTTCCGGGGGGA \\
\hline UC-rev & TCCCCCCGGAAGACGTGCTGGCAG \\
\hline AA-for & CTGCCAGCACGAATTCCGGGGGGA \\
\hline AA-rev & TCCCCCCGGAATTCGTGCTGGCAG \\
\hline AU-for & CTGCCAGCACGATTTCCGGGGGGA \\
\hline AU-rev & TCCCCCCGGAAATCGTGCTGGCAG \\
\hline AC-for & CTGCCAGCACGACTTCCGGGGGGA \\
\hline AC-rev & TCCCCCCGGAAGTCGTGCTGGCAG \\
\hline CG-for & CTGCCAGCACGCGTTCCGGGGGGA \\
\hline CG-rev & TCCCCCCGGAACGCGTGCTGGCAG \\
\hline GG-for & CTGCCAGCACGGGTTCCGGGGGGA \\
\hline GG-rev & TCCCCCCGGAACCCGTGCTGGCAG \\
\hline UG-for & CTGCCAGCACGTGTTCCGGGGGGA \\
\hline UG-rev & TCCCCCCGGAACACGTGCTGGCAG \\
\hline
\end{tabular}


A

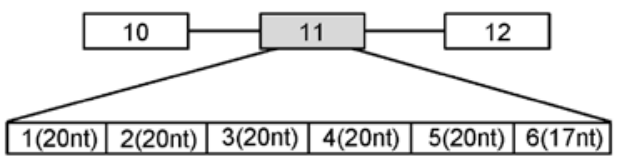

$\Delta 11-1$

$\Delta 11-2$

$\Delta 11-3$

$\Delta 11-4$

$\Delta 11-5$

$\Delta 11-6$

B
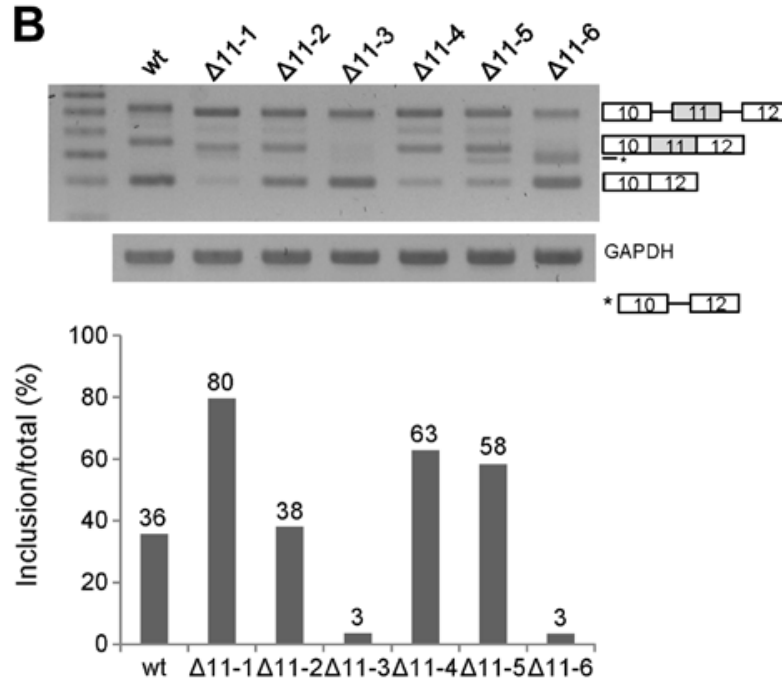

Figure 1. Exon 11 contains various regulatory elements for exon 11 inclusion of Ron pre-mRNA. (A) A series of deletion mutants $(\Delta 11-1, \Delta 11-2, \Delta 11-3$, $\Delta 11-4, \Delta 11-5$ and $\Delta 11-6)$ is shown. The lengths of each section are indicated. Dotted lines indicate the deleted sections for each mutant. (B) RT-PCR analysis of wild-type (wt) minigene and deletion mutants. The partially spliced product is marked with a star. GAPDH was used as a control. Quantitation of the results is shown as a ratio of exon 11 inclusion to total RNA.

wild-type. However, exon 11 inclusion was increased significantly in the $\Delta 11-1 \Delta 11-4$ and $\Delta 11-5$ mutants $(\sim 44, \sim 27$ and $\sim 22 \%$ each). In addition, the $\Delta 11-3$ and $\Delta 11-6$ mutants showed decreased exon 11 inclusion ( $\sim 33$ and $\sim 33 \%$ each). Thus, we concluded that the RNA length $(20 \mathrm{nt})$ and the sequences of 11-3 and 11-6 contained an enhancer for exon 11 inclusion, whereas 11-1, 11-4 and 11-5 RNA contained an inhibitor for exon 11 inclusion of Ron pre-mRNA. Since the $\Delta 11-6$ mutant produced a product caused by partial splicing (shown as *), the 11-6 RNA part probably also regulated the partial splicing. To further identify the enhancer for exon 11 inclusion, we selected the 11-3 RNA part for further study.

The 2nd 10 nt but not the 1 st 10 nt in 11-3 functions as an enhancer for exon 11 inclusion. To further identify the enhancer for exon 11 inclusion in 11-3 RNA of exon 11, we dissected 20 nt of 11-3 RNA into two 10-nt RNA sections. The two 10-nt deleted mutants were produced as shown in Fig. 2A, labeled $\Delta 11-3-1$ and $\Delta 11-3-2$. RT-PCR analysis showed that only the $\Delta 11-3-2$ mutant decreased exon 11 inclusion $(\sim 35 \%)$ whereas $\Delta 11-3-1$ increased exon 11 inclusion ( 21\%) (Fig. 2B).
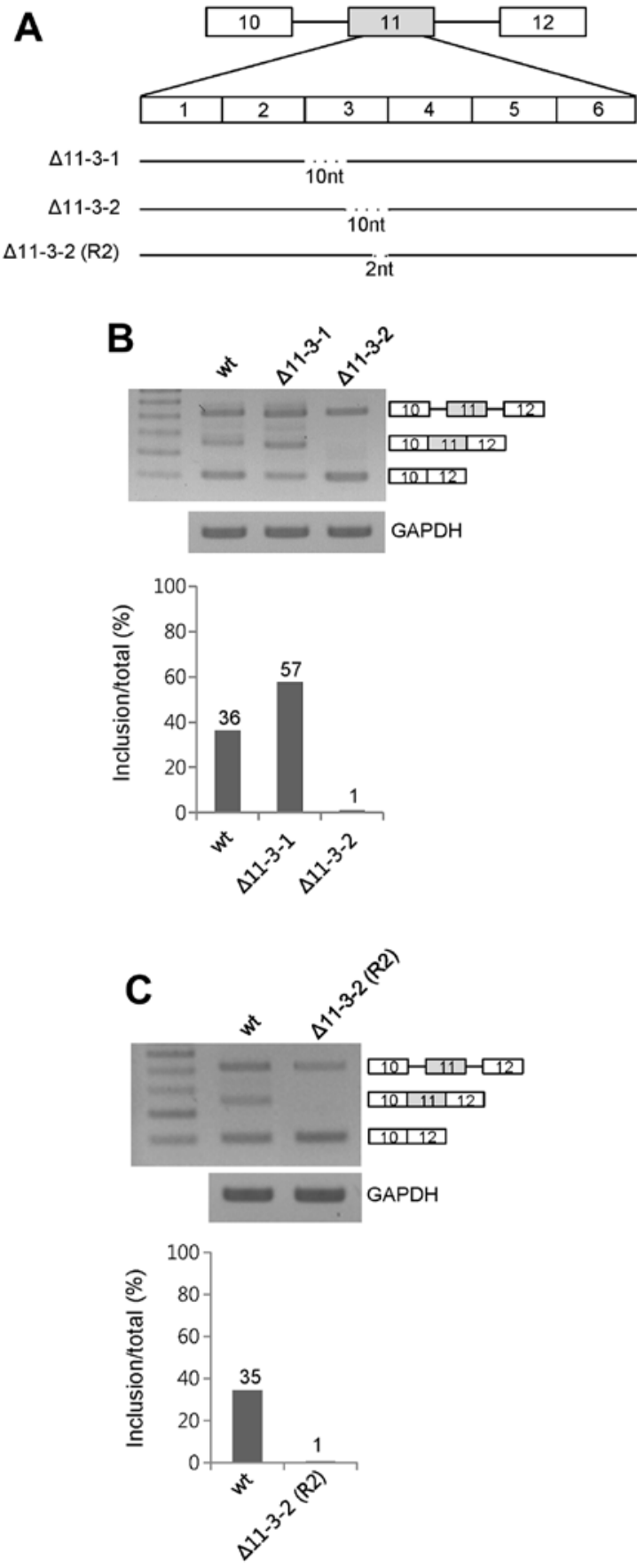

Figure 2. The $2 \mathrm{nd} 10 \mathrm{nt}$ but not the $1 \mathrm{st} 10 \mathrm{nt}$ in 11-3 functions as an enhancer for exon 11 inclusion. (A) Deletion mutants ( $\Delta 11-3-1$ and $\Delta 11-3-2)$ and [ $\Delta 11$ 3-2(R2)] are shown. The 10-nt and 2-nt RNAs were deleted in each mutant. (B and C) RT-PCR analysis of wild-type (wt) minigene and deletion mutants. Quantitation of the results is shown as the ratio of exon 11 inclusion to total RNA.

Therefore, we conclude that $10 \mathrm{nt}$ of the 11-3-2 RNA includes the enhancer for exon 11 inclusion.

The 2-nt RNA at the 3' end of the 11-3-2 RNA functions as an enhancer for exon 11 inclusion. To further understand the enhancer for exon 11 inclusion, we dissected the 2nd 10-nt RNA of 11-3. We deleted $2 \mathrm{nt}$ from the 3 ' end of the 10-nt RNA, labeled $\Delta 11-3-2$ (R2) (Fig. 2A). After extraction of RNA from the minigene-transfected cells, we performed RT-PCR. The results in Fig. 3B demonstrated that the $\Delta 11-3-2$ (R2) mutant 
A
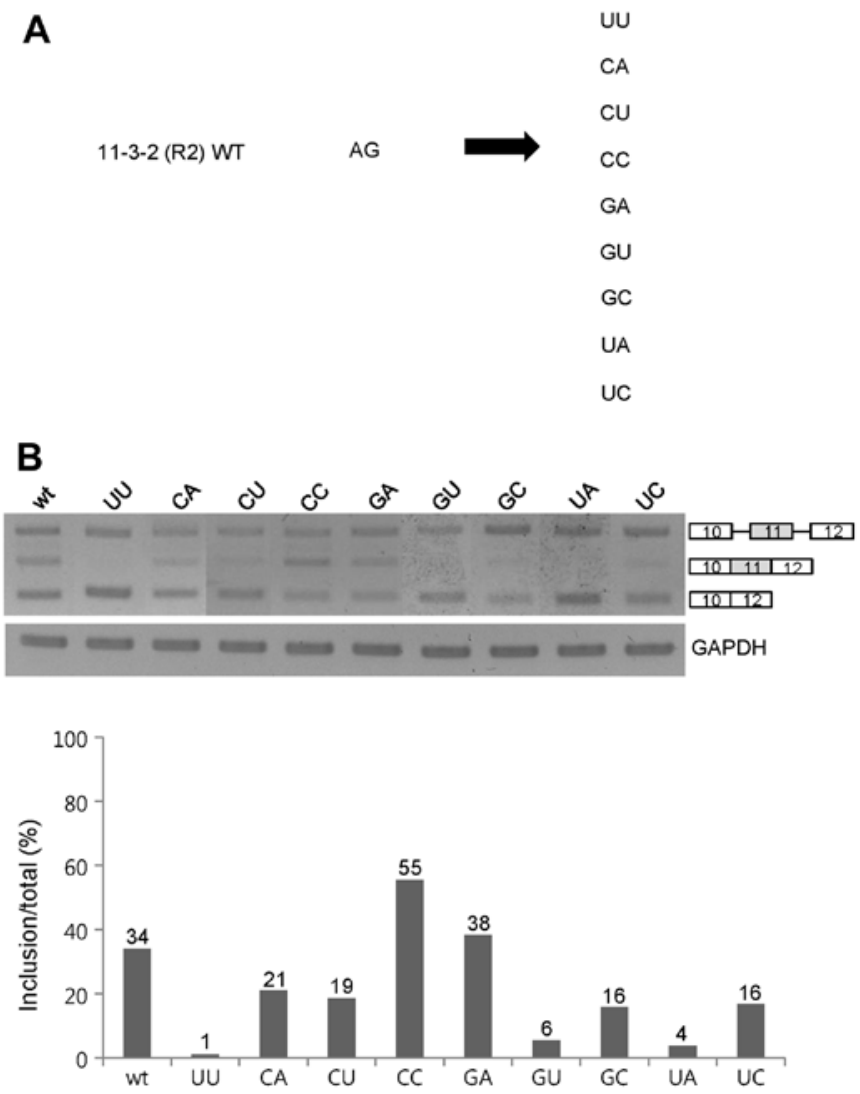

Figure 3. GA, CC and the wild-type AG sequences function as enhancers for exon 11 inclusion of Ron pre-mRNA. (A) Both nucleotides of AG [113-2 (R2)] were mutated to other sequences. The mutated RNA sequences in each mutant are shown. (B) RT-PCR analysis of wild-type (wt) minigene and mutants. Quantitation of the results of RT-PCR is shown.

expressed the exon 11 skipped form exclusively (Fig. 2C). Therefore, we concluded that the 2-nt RNA at the $3^{\prime}$ end of the 11-3-2 RNA contains enhancers for exon 11 inclusion.

GA, CC and the wild-type AG sequences function as enhancers for exon 11 inclusion of Ron pre-mRNA. Since the 2-nt RNA at the 11-3-2 section on exon 11 acts as a strong enhancer for exon 11 inclusion, we decided to pinpoint the sequence requirements for the 2-nt RNA. As the first approach, we performed substitution mutagenesis analysis on both nucleotides of the 2-nt RNA. We mutated the AG sequence into various sequences that cover all of the four base combinations (Fig. 3A). Among the mutants, as shown in Fig. 3B, exon 11 inclusion was completely compromised in the UU, GU and UA mutants. The CA, CU, GC and UC mutants showed a significant decrease in exon 11 inclusions $(\sim 13, \sim 15, \sim 18$ and $\sim 18 \%)$. However, exon 11 inclusion was increased in the CC mutant, whereas the GA mutant showed a comparable level of exon 11 inclusion as the wild-type minigene. The substitution mutant results indicate that most 2-nt sequences promoted exon 11 skipping of Ron premRNA. One opposite case was the CC RNA sequence that promoted exon 11 inclusion $(21 \%)$. Therefore, we concluded that the GA, CC and AG sequences at the 3' end of 11-3-2 RNA are required for the function of the 2-nt RNA as an enhancer for exon 11 inclusion of Ron pre-mRNA.
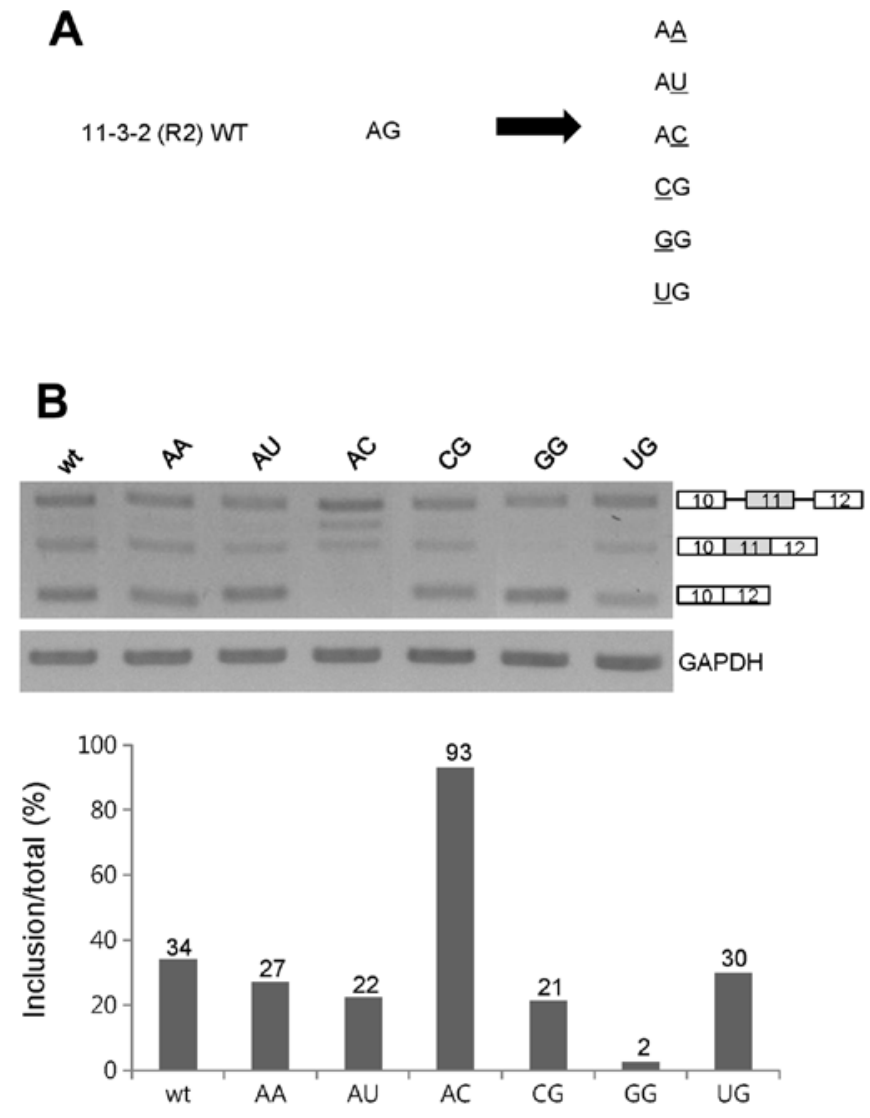

Figure 4. UG and AC also function as enhancers for exon 11 inclusion of Ron pre-mRNA. (A) The $1 \mathrm{nt}$ of AG was mutated in each mutant. The mutated RNA sequences in each mutant are shown. (B) RT-PCR analysis of wild-type (wt)minigene and mutants. Quantitation of the results of RT-PCR is shown.

$U G$ and $A C$ also function as enhancers for exon 11 inclusion of Ron pre-mRNA. In order to further understand the sequence requirement of the 2-nt enhancer at the $3^{\prime}$ end of the 11-3-2 RNA, as the second approach, we performed single nucleotide substitution mutagenesis analysis. In the first set of single nucleotide mutagenesis assay, we mutated the A nucleotide of wild-type AG at the 11-3-2 (R2) RNA into the C, G or U nucleotide, whereas the $G$ residue at the wild-type $A G$ remain unchanged, and were named as CG, 1GG and UG. In the second set of mutagenesis, we mutated the $\mathrm{G}$ nucleotide of the AG dinucleotide into A, U and $\mathrm{C}$ nucleotides separately, whereas the $\mathrm{A}$ residue of $\mathrm{AG}$ remained unchanged, and were named as AA, AU and AC (Fig. 4A). RT-PCR analysis in Fig. 4B shows that the GG mutant demonstrated a completely compromised exon 11 inclusion. In addition, exon 11 inclusion was significantly reduced in the CG and UG mutant $(\sim 13 \%)$. In contrast, exon 11 skipping was not significantly decreased in the UG mutant $(\sim 3 \%)$. Thus, to maintain the enhancer function of the wild-type AG dinucleotide for exon 11 inclusion, the first position should be $A$ and $U$ but not $C$ and $G$ residue. In the second residue substitution mutants, as shown in Fig. 4B, the $\mathrm{AC}$ mutant, in which the $\mathrm{G}$ nucleotide of the $\mathrm{AG}$ dinucleotide was substituted by the $\mathrm{C}$ residue, showed the exon 11 inclusion form exclusively ( 93\%). Thus, the AC sequence functions as a stronger enhancer. However, exon 11 inclusion was reduced in the AU and AA mutants ( 12 and $~ 7 \%)$. Therefore, we 
conclude that the $\mathrm{G}$ or $\mathrm{C}$ nucleotide at the second position of the AG dinucleotide maintains or increases its enhancer function for exon 11 inclusion. Collectively, the $\mathrm{A}$ or $\mathrm{U}$ residue at the first position, in combination with $\mathrm{G}$ or $\mathrm{C}$ at the second position of the wild-type AG dinucleotide are required for the enhancer function for exon 11 inclusion. We summarize that UG and AC function as enhancers for exon 11 inclusion of Ron pre-mRNA.

\section{Discussion}

Ron proto-oncogene, a receptor tyrosine kinase, produces the $\Delta 165$ isoform through exon 11 skipping. The $\Delta 165$ isoform is a constitutively active isoform without the binding of the MSP ligand. Exon 11 inclusion is regulated by the juxtaposed enhancers and inhibitors which are located at exon 12 . We identified a 2-nt enhancer for exon 11 inclusion at exon 11, located at $74 \mathrm{nt}$ upstream from the $5^{\prime}$ splice site of exon 11 , through serial deletion analysis. Through double base substitution analysis, we demonstrated that, in addition to the AG sequence, $\mathrm{GA}$ and $\mathrm{CC}$ also maintained their enhancer function. Furthermore, through the single base substitution analysis, we found that UG and AC function as enhancers for exon 11 inclusion of Ron pre-mRNA.

Exon 11 inclusion/skipping is regulated by multiple cis-acting elements. Previously, it was shown that an enhancer is located at exon 12 to promote exon 11 skipping. It was also shown that the inhibitor RNA, which is located next to the enhancer, promotes exon 11 inclusion. Most importantly, the antagonistic effects of the enhancer and inhibitor regulate exon 11 inclusion and skipping. Our results here demonstrated that exon 11 inclusion/skipping is regulated by, in addition to the enhancer and inhibitor on exon 12, the enhancer on exon 11. Through multiple 20-nt deletion analyses, we found that different 20-nt deletions had different effects on exon 11 splicing.

Exon 11 inclusion was increased significantly in the $\Delta 11-1$, $\Delta 11-4$ and $\Delta 11-5$ mutants, was decreased significantly in the $\Delta 11-3$ and $\Delta 11-6$ mutants, and remained at a similar level for the wild-type minigene in the $\Delta 11-2$ mutant. Our results indicate that most deletion mutations of exon 11 showed the alteration of exon 11 inclusion. Thus, we concluded that exon inclusion/skipping is regulated by multiple $c i s$-elements.

Simple determination of the exon enhancer by deletion mutagenesis is not always correct. Our deletion mutagenesis analysis showed that the 20-nt RNA (11-3) had the enhancer function (Fig. 1B). However, through further deletion we found that the upstream $10 \mathrm{nt}$ had an inhibitor function, whereas the downstream $10 \mathrm{nt}$ functioned as an enhancer (Fig. 2B). Thus, it is not correct to determine the splicing enhancer by deletion mutagenesis, although deletion mutagenesis definitely provides important information. cis-acting elements of premRNA splicing are composed of different combinations of four nucleotides, and usually provide the functional targets for trans-acting elements. It is not surprising that each 10-nt RNA had the opposite functions on exon 11 inclusion. One possibility is that one 10-nt RNA section provided the contact for activator proteins, and the other one provided the contact for the inhibitory proteins. Another possibility is that the dele- tion of $10 \mathrm{nt}$ made the flanking sequences to be connected to produce another enhancer sequence. Therefore, determination of splicing enhancer by deletion mutagenesis is not always correct.

Length and sequence of RNA play roles in exon 11 inclusion/ skipping. Our substitution analysis of the AG dinucleotides demonstrated that different bases had different effects on exon 11 inclusion of Ron pre-mRNA. By double nucleotide mutagenesis, we found that the GA, CC as well as AG sequences at the 3' end of the 11-3-2 RNA were required for the function of the 2-nt RNA as an enhancer for exon 11 inclusion of Ron pre-mRNA. By single base substitution analysis, we found that the $\mathrm{A}$ or $\mathrm{U}$ residue at the first position, and the $\mathrm{G}$ or $\mathrm{C}$ at the second position of the AG dinucleotide were required for the enhancer function for exon 11 inclusion. Surprisingly, we found that several mutants (UA, GC, UU and GG) completely destroyed exon 11 inclusion, whereas the AC mutant completely destroyed exon 11 skipping. Our results indicate the high sequence requirement of the enhancer for exon 11 inclusion of Ron pre-mRNA.

\section{Acknowledgements}

The present study was supported by the Mid-Career Researcher Program through a National Research Foundation (NRF) grant (2013029711) funded by the Ministry of Education, Science, and Technology (MEST), Korea; and a Systems Biology Infrastructure Establishment grant provided by the Gwangju Institute of Science and Technology (GIST) in 2013.

\section{References}

1. Ronsin C, Muscatelli F, Mattei MG and Breathnach R: A novel putative receptor protein tyrosine kinase of the met family. Oncogene 8: 1195-1202, 1993.

2. Camp ER, Liu W, Fan F, Yang A, Somcio R and Ellis LM: RON, a tyrosine kinase receptor involved in tumor progression and metastasis. Ann Surg Oncol 12: 273-281, 2005.

3. Gaudino G, Follenzi A, Naldini L, et al: RON is a heterodimeric tyrosine kinase receptor activated by the HGF homologue MSP. EMBO J 13: 3524-3532, 1994.

4. Angeloni D, Danilkovitch-Miagkova A, Ivanov SV, et al: Gene structure of the human receptor tyrosine kinase RON and mutation analysis in lung cancer samples. Genes Chromosomes Cancer 29: 147-156, 2000.

5. Follenzi A, Bakovic S, Gual P, Stella MC, Longati P and Comoglio PM: Cross-talk between the proto-oncogenes Met and Ron. Oncogene 19: 3041-3049, 2000.

6. Wang MH, Ronsin C, Gesnel MC, et al: Identification of the ron gene product as the receptor for the human macrophage stimulating protein. Science 266: 117-119, 1994.

7. Danilkovitch A and Leonard EJ: Kinases involved in MSP/RON signaling. J Leukoc Biol 65: 345-348, 1999.

8. Longati P, Bardelli A, Ponzetto C, Naldini L and Comoglio PM: Tyrosines1234-1235 are critical for activation of the tyrosine kinase encoded by the MET proto-oncogene (HGF receptor). Oncogene 9: 49-57, 1994.

9. Iwama A, Yamaguchi N and Suda T: STK/RON receptor tyrosine kinase mediates both apoptotic and growth signals via the multifunctional docking site conserved among the HGF receptor family. EMBO J 15: 5866-5875, 1996.

10. Danilkovitch-Miagkova A: Oncogenic signaling pathways activated by RON receptor tyrosine kinase. Curr Cancer Drug Targets 3: 31-40, 2003.

11. Chen Q, Seol DW, Carr B and Zarnegar R: Co-expression and regulation of Met and Ron proto-oncogenes in human hepatocellular carcinoma tissues and cell lines. Hepatology 26: 59-66, 1997. 
12. Maggiora P, Marchio S, Stella MC, et al: Overexpression of the RON gene in human breast carcinoma. Oncogene 16: 2927-2933 1998.

13. Wang MH, Yao HP and Zhou YQ: Oncogenesis of RON receptor tyrosine kinase: a molecular target for malignant epithelial cancers. Acta Pharmacol Sin 27: 641-650, 2006.

14. Lu Y, Yao HP and Wang MH: Multiple variants of the RON receptor tyrosine kinase: biochemical properties, tumorigenic activities, and potential drug targets. Cancer Lett 257: 157-164, 2007.

15. Collesi C, Santoro MM, Gaudino G and Comoglio PM: A splicing variant of the RON transcript induces constitutive tyrosine kinase activity and an invasive phenotype. Mol Cell Biol 16: 5518-5526, 1996.

16. Ghigna C, Giordano S, Shen H, et al: Cell motility is controlled by SF2/ASF through alternative splicing of the Ron protooncogene. Mol Cell 20: 881-890, 2005.

17. Wahl MC, Will CL and Luhrmann R: The spliceosome: design principles of a dynamic RNP machine. Cell 136 701-718, 2009.

18. Moore MJ and Proudfoot NJ: Pre-mRNA processing reaches back to transcription and ahead to translation. Cell 136: 688-700, 2009.

19. Han J, Xiong J, Wang D and Fu XD: Pre-mRNA splicing: where and when in the nucleus. Trends Cell Biol 21: 336-343, 2011.
20. Black DL: Mechanisms of alternative pre-messenger RNA splicing. Annu Rev Biochem 72: 291-336, 2003.

21. Blencowe BJ: Alternative splicing: new insights from global analyses. Cell 126: 37-47, 2006.

22. Keren H, Lev-Maor G and Ast G: Alternative splicing and evolution: diversification, exon definition and function. Nat Rev Genet 11: 345-355, 2010.

23. Grabowski PJ and Black DL: Alternative RNA splicing in the nervous system. Prog Neurobiol 65: 289-308, 2001.

24. David CJ and Manley JL: Alternative pre-mRNA splicing regulation in cancer: pathways and programs unhinged. Genes Dev 24: $2343-2364,2010$

25. Lee J,Zhou J,Zheng X, et al: Identification of a novel cis-element that regulates alternative splicing of Bcl-x pre-mRNA. Biochem Biophys Res Commun 420: 467-472, 2012.

26. Cho S, Moon H, Yang X, et al: Validation of trans-acting elements that promote exon 7 skipping of SMN2 in SMN2-GFP stable cell line. Biochem Biophys Res Commun 423: 531-535, 2012.

27. Kan JL and Green MR: Pre-mRNA splicing of IgM exons M1 and M2 is directed by a juxtaposed splicing enhancer and inhibitor. Genes Dev 13: 462-471, 1999.

28. Shen H, Kan JL, Ghigna C, Biamonti G and Green MR: A single polypyrimidine tract binding protein (PTB) binding site mediates splicing inhibition at mouse IgM exons M1 and M2. RNA 10: 787-794, 2004. 